\title{
Tendance liées à l'utilisation des antimicrobiens et la résistance aux antimicrobiens au Canada : 2014
}

\author{
Ebrahim $\mathrm{M}^{1}$, Gravel $\mathrm{D}^{1 *}$, Thabet $\mathrm{C}^{1}$, Abdesselam $\mathrm{K}^{1}$, Paramalingam $\mathrm{S}^{1}$, Hyson $\mathrm{C}^{1}$
}

\section{Résumé}

Contexte : Sur la scène mondiale, on craint que l'émergence de la résistance aux antimicrobiens menace notre capacité à traiter les maladies infectieuses. Le Système canadien de surveillance de la résistance aux antimicrobiens (SCSRA) a été créé en réponse à l'engagement du gouvernement du Canada en matière de lutte contre la résistance aux antimicrobiens. Le SCSRA intègre l'information provenant de neuf différents systèmes de surveillance nationaux pour faire le suivi de l'utilisation des antimicrobiens et de la résistance aux antimicrobiens à la fois chez les humains et chez les animaux afin d'éclairer la recherche et les politiques en matière d'utilisation des antimicrobiens et de la résistance aux antimicrobiens.

Objectif : Mettre en lumière les données du SCSRA sur l'utilisation des antimicrobiens chez les humains et les animaux, les tendances relatives à la résistance aux antimicrobiens dans les infections humaines aussi bien dans les milieux hospitaliers que dans les milieux communautaires ainsi que les bactéries relatives à la résistance aux antimicrobiens trouvées chez les animaux destinés à la production alimentaire.

Méthodologie : L'information au sujet de l'utilisation des antimicrobiens chez les humains et les animaux est achetée et les renseignements additionanels sur l'utilisation chez les animaux sont recueillis par l'entremise du Programme intégré canadien de surveillance de la résistance aux antimicrobiens (PICRA). Les données sur la résistance aux antimicrobiens chez les humains se concentrent sur les organismes ayant priorité absolue. Les données sur la résistance aux antimicrobiens des organismes prioritaires en milieu hospitalier sont recueillies par l'intermédiaire du Programme canadien de surveillance des infections nosocomiales (PCSIN), du Système canadien de surveillance des laboratoires de tuberculose (SCSLT), du Système canadien de déclaration des cas de tuberculose (SCDCT), et du PICRA. Les données sur la résistance aux antimicrobiens en milieu communautaire sont recueillies par l'intermédiaire du SCSLT, du SCDCT, du PICRA, du Système de surveillance de la résistance aux antimicrobiens de Neisseria gonorrhoeae (SSRANG) ainsi que de la surveillance à l'échelle nationale des maladies invasives à streptocoque (SNMIS). Les données sur la résistance aux antimicrobiens chez les animaux sont recueillies par l'entremise du PICRA.

Résultats : En ce qui a trait à l'utilisation d'antibiotiques en 2014, environ $82 \%$ des antimicrobiens étaient distributés aux animaux destinés à la production alimentaire, $18 \%$ aux humains, et moins de un pour cent aux animaux de compagnie et aux cultures. Au cours des cinq dernières années, $73 \%$ des antimicrobiens distribués aux animaux destinés à la production alimentaire appartenaient aux mêmes catégories que celles utilisées en médecine humaine. L'utilisation d'antibiotiques chez les humains est demeurée relativement stable. Les tendances en 2014 pour la résistance aux antimicrobiens dans les hôpitaux comprennent une baisse des taux d'infection nosocomiale à Clostridium difficile acquise dans les hôpitaux résistant aux antimicrobiens à 3,4 cas pour 1000 hospitalisations, des taux d'infection à Staphylococcus aureus résistant à la méthicilline (SARM) à 2,89 cas pour 10000 jours-patients, ainsi que des taux d'infection à entérocoques résistants à la vancomycine $(E R V)$ à 0,45 cas pour 10000 jours-patients. La résistance à un certain nombre d'antimicrobiens utilisés pour traiter Streptococcus pneumoniae a diminué depuis l'introduction du vaccin contre le pneumocoque en 2010. En revanche, les tendances en 2014 pour la résistance aux antimicrobiens au sein de la collectivité comprenaient la hausse des taux de N. gonorrhoeae contractées en milieu communautaire - 52,4\% des isolats étaient résistants à au moins un antibiotique. Les tendances pour les entérobactéries résistantes aux carbapénèmes (ERC) étaient stables à 0,22 cas pour 10000 jours-patients. De plus, de 2004 à 2014, neuf pour cent des cas de tuberculose (TB) à culture positive étaient résistants à au moins un antituberculeux de première intention, et cette tendance est demeurée relativement stable au cours de cette période. Les tendances en 2014 pour la résistance aux antimicrobiens chez les animaux destinés à la production alimentaire ont indiquée une diminution de la résistance aux céphalosporines (ceftriaxone) de troisième génération des espèces Escherichia coli et Salmonella chez la volaille associée à une diminution de l'utilisation des céphalosporines dans les fermes d'élevage de poulets, mais la résistance à la ciprofloxacine des espèces Campylobacter chez les poulets et les boivins a augmenté.

Conclusion : De façon générale, I'utilisation d'antibiotiques chez les humains n'a pas diminué en dépit des préoccupations au sujet d'une utilisation excessive. Bien que les taux de résistance de $C$. difficile, d'ERV, de SARM et de $S$. pneumoniae résistant aux antimicrobiens aient progressivement diminué et que la tuberculose pharmacorésistante et les ERC soient demeurées stables, le taux d'infection à $N$. gonorrhoeae résistante aux médicaments en milieu communautaire a augmenté. Bien que les efforts visant à réduire l'utilisation des antibiotiques chez les animaux aient été atteints avec un certain succès, la résistance aux antimicrobiens continue d'avoir des taux assez élevés chez les animaux destinés à la production alimentaire.

\section{Affiliation}

${ }^{1}$ Centre de la lutte contre les maladies transmissibles et les infections, Agence de la santé publique du Canada, Ottawa (Ontario)

*Correspondance : denise. graveltropper@phac-aspc.gc.ca 
Citation proposée : Ebrahim M, Gravel D, Thabet C, Abdesselam K, Paramalingam S, Hyson C. Tendances liées à l'utilisation des antimicrobiens et à la résistance aux antimicrobiens au Canada : 2014. Relevé des maladies transmissibles au Canada 2016;42(11):251-6.

https://doi.org/10.14745/ccdr.v42i11a02f

\section{Introduction}

La portée de l'utilisation des antimicrobiens et de la résistance aux antimicrobiens sont des préoccupations d'ordre mondial en raison des niveaux de résistance accrus détectés dans certains microbes pathogènes et de la pression sélective découlant de l'utilisation des antimicrobiens tant chez les animaux que chez les humains (1). Les effets préjudiciables de la résistance aux antimicrobiens se manifestent déjà dans le monde entier, et les infections résistantes aux antimicrobiens coûtent la vie à au moins 50000 personnes chaque année en Europe et aux États-Unis seulement, en plus des centaines de milliers de personnes qui meurent également dans d'autres régions du monde (2). En vue de surveiller l'utilisation des antimicrobiens et la résistance aux antimicrobiens au Canada, on a créé le Système canadien de surveillance de la résistance aux antimicrobiens (SCSRA). Grâce à la collaboration avec des intervenants internes et externes, le SCSRA intègre plusieurs sources d'information de surveillance au sujet de l'utilisation des antimicrobiens et de la résistance à ceux-ci, en vue de fournir un portrait national unifié de l'utilisation des antimicrobiens et de la résistance aux antimicrobiens chez les humains et les animaux au Canada. Les données sur les humains et les animaux sont incluses, car l'utilisation inappropriée des antimicrobiens chez les animaux destinés à la production alimentaire est un problème de santé publique, étant donné qu'elle contribue à l'apparition de bactéries résistantes chez les animaux qui peuvent être transmises aux humains par l'approvisionnement alimentaire (3). Le premier rapport du SCSRA publié en mars 2015 présentait les données sur I'utilisation et la résistance aux antimicrobiens au Canada jusqu'en 2013 (4).

L'objectif de cette synthèse est de mettre en évidence les principales constatations du deuxième rapport du SCSRA résumant les données de surveillance jusqu'en 2014. Le rapport complet est disponible en ligne (5).

\section{Méthodologie}

L'information est recueillie dans quatre domaines clés: I'utilisation des antimicrobiens chez les humains, I'utilisation des antimicrobiens chez les animaux, la résistance aux antimicrobiens chez les humains dans les milieux de soins de santé et communautaires, et la résistance aux antimicrobiens dans les animaux destinés à la production alimentaire.

\section{Utilisation des antimicrobiens chez les humains et les animaux}

Le SCRSA achète des données sur l'utilisation des antimicrobiens chez les humains auprès d'IMS Health Canada Inc., une entreprise mondiale qui recueille de l'information sur les achats d'antibiotiques dans les hôpitaux et sur les ordonnances délivrées par les pharmacies de détail dans la collectivité, ainsi que sur les antimicrobiens prescrits par les médecins. Le SCSRA obtient des données sur l'utilisation des antimicrobiens chez les animaux auprès de l'Institut canadien de la santé animale, qui recueille des données concernant plus de $90 \%$ de l'ensemble des ventes de produits pharmaceutiques vétérinaires homologués au Canada. L'Agence de la santé publique du Canada (ASPC) recueille des renseignements supplémentaires sur l'utilisation des antimicrobiens auprès de fermes sentinelles par l'entremise du Programme intégré canadien de surveillance de la résistance aux antimicrobiens
(PICRA). Le PICRA surveille les tendances en matière d'utilisation des antimicrobiens et de résistance aux antimicrobiens chez certains organismes bactériens isolés de sources humaines, animales et alimentaires de partout au Canada (6).

\section{Résistance aux antimicrobiens chez les humains}

\section{Milieux de soins}

Le SCSRA recueille des données concernant la résistance aux antimicrobiens dans les organismes prioritaires transmis dans les milieux de soins de santé grâce à un certain nombre de différents systèmes de surveillance. Le Programme canadien de surveillance des infections nosocomiales (PCSIN) assure le suivi de C. difficile, des organismes producteurs de carbapénémases (OPC), et des entérobactéries résistantes aux carbapénèmes (ERC), des Staphylococcus aureus résistants à la méthicilline (SARM), et des entérocoques résistants à la vancomycine (ERV) (7). Les isolats humains de Salmonella dans les hôpitaux sont pris en compte dans le cadre du PICRA (8). Les données sur les cas de tuberculose dans les hôpitaux sont fournies par l'entremise du Système canadien de surveillance des laboratoires de tuberculose (SCSLT) et du Système canadien de déclaration des cas de tuberculose (SCDCT) (9).

\section{Milieux communautaires}

La résistance aux antimicrobiens est surveillée en vue de détecter les organismes prioritaires suivants, transmis principalement dans des milieux communautaires: Streptococcus pneumoniae, le streptocoque du groupe A, Neisseria gonorrhoeae et Mycobacterium tuberculosis. Les laboratoires provinciaux de santé publique soumettent des isolats au Laboratoire national de microbiologie (LNM) pour le sérotypage de Streptococcus et de Neisseria gonorrhoeae. Les données relatives à la résistance aux antimicrobiens concernant Mycobacterium tuberculosis sont transmises à l'ASPC par l'intermédiaire du SCSLT et du SCDCT (9).

\section{Résistance aux antimicrobiens chez les animaux destinés à la production alimentaire}

Le PICRA comprend des tests de sensibilité pour les bactéries prioritaires suivantes transmises aux humains par les animaux destinés à la production alimentaire : les espèces Escherichia coli, Campylobacter et Salmonella (8). Les échantillons d'E. coli sont recueillis à trois endroits différents dans la chaîne alimentaire (fermes, abattoirs et magasins de détail) pour le poulet et le porc, et dans des abattoirs ou des magasins de détail pour les bovins. Des échantillons de Campylobacter sont recueillis tout au long de la chaîne alimentaire pour le poulet et uniquement dans des abattoirs pour les porcs et les bovins; des échantillons de l'espèce Salmonella sont prélevés dans des fermes et des abattoirs pour le poulet (5).

Une synthèse de toutes les données recueillies par le Système canadien de surveillance au sujet de la résistance aux antimicrobiens des organismes prioritaires pour les humains et les animaux est résumée dans le tableau 1. 
Tableau 1 : Sources de données sur la résistance aux antimicrobiens chez les humains et les animaux fournies au Système canadien de surveillance de la résistance aux antimicrobiens

\begin{tabular}{|c|c|c|c|}
\hline $\begin{array}{l}\text { Données } \\
\text { recueillies }\end{array}$ & $\begin{array}{l}\text { Système de } \\
\text { surveillance }\end{array}$ & Agents pathogènes & $\begin{array}{l}\text { Fournit de } \\
\text { I'information } \\
\text { pour: }\end{array}$ \\
\hline \multirow[t]{3}{*}{$\begin{array}{l}\text { Résistance aux } \\
\text { antimicrobiens } \\
\text { dans les } \\
\text { hôpitaux }\end{array}$} & PCSIN & $\begin{array}{l}\text { - Staphylococcus aureus } \\
\text { résistant à la méthicilline } \\
\text { - Clostridium difficile } \\
\text { - Entérocoques résistants à la } \\
\text { vancomycine } \\
\text { - Entérobactéries résistantes } \\
\text { aux carbapénèmes (ERC) et } \\
\text { organismes producteurs de } \\
\text { carbapénémases (OPC) : } \\
\text { - Espèce Acinetobacter } \\
\text { producteur de } \\
\text { carbapénémase (APC) } \\
\text { - Entérobactéries } \\
\text { productrices de } \\
\text { carbapénémase (EPC) }\end{array}$ & $\begin{array}{l}\text { - Infections associées } \\
\text { aux soins de } \\
\text { santé et au milieu } \\
\text { communautaire } \\
\text { dans les hôpitaux } \\
\text { de soins de courte } \\
\text { durée }\end{array}$ \\
\hline & $\begin{array}{l}\text { SCSLT et } \\
\text { SCDCT }\end{array}$ & - Mycobacterium tuberculosis & $\begin{array}{l}\text { - Profils de } \\
\text { pharmacorésistance }\end{array}$ \\
\hline & PICRA & - Salmonella & - Cas à I'hôpital \\
\hline \multirow{4}{*}{$\begin{array}{l}\text { Résistance aux } \\
\text { antimicrobiens } \\
\text { en milieu } \\
\text { communautaire }\end{array}$} & $\begin{array}{l}\text { SCSLT et } \\
\text { SCDCT }\end{array}$ & - Mycobacterium tuberculosis & $\begin{array}{l}\text { - Profils de } \\
\text { pharmacorésistance }\end{array}$ \\
\hline & SSRANG & - Neisseria gonorrhoeae & $\begin{array}{l}\text { - Sensibilité aux } \\
\text { antimicrobiens }\end{array}$ \\
\hline & PICRA & - Salmonella & $\begin{array}{l}\text { - Cas } \\
\text { communautaires }\end{array}$ \\
\hline & SNMIS & $\begin{array}{l}\text { - Streptococcus pneumoniae } \\
\text { - Streptococcus pyogenes } \\
\text { - Streptococcus agalactiae }\end{array}$ & $\begin{array}{l}\text { - Sensibilité aux } \\
\text { antimicrobiens }\end{array}$ \\
\hline $\begin{array}{l}\text { Résistance aux } \\
\text { antimicrobiens } \\
\text { chez les } \\
\text { animaux } \\
\text { destinés à la } \\
\text { production } \\
\text { animale }\end{array}$ & PICRA & $\begin{array}{l}\text { - E. coli } \\
\text { - Campylobacter } \\
\text { - Espèce Salmonella }\end{array}$ & $\begin{array}{l}\text { - Résistance chez les } \\
\text { bactéries d'origine } \\
\text { alimentaire dans le } \\
\text { poulet, le porc et les } \\
\text { bovins }\end{array}$ \\
\hline \multicolumn{4}{|c|}{$\begin{array}{l}\text { Abréviations : SSRANG, Système de surveillance de la résistance aux antimicrobiens de } \\
\text { Neisseria gonorrhoeae; PICRA, Programme intégré canadien de surveillance de la résistance } \\
\text { aux antimicrobiens; PCSIN, Programme canadien de surveillance des infections nosocomiales; } \\
\text { surveillance des laboratoires de tuberculose; SCSLT, Système canadien de surveillance des } \\
\text { laboratoires de tuberculose; SCDCT, Système canadien de déclaration des cas de tuberculose; } \\
\text { SNMIS, surveillance à l'échelle nationale des maladies invasives à streptocoque }\end{array}$} \\
\hline
\end{tabular}

\section{Analyse}

En raison de la nature de la méthode utilisée pour recueillir les données issues des systèmes de surveillance établis, les analyses statistiques sont descriptives. Les nombreuses années de données ont facilité l'établissement de points de repère et l'analyse des tendances au fil du temps.

\section{Résultats}

\section{Utilisation des antimicrobiens}

Environ 1,4 million de kilogrammes $(\mathrm{kg}) \mathrm{d}$ 'antimicrobiens importants sur le plan médical ont été distribués et/ou vendus en 2014. Environ $82 \%$ des antimicrobiens ont été utilisés chez des animaux destinés à la production alimentaire, $18 \%$ chez les humains et moins de un pour cent pour les animaux de compagnie et les cultures. Chez les humains, les classes prédominantes d'antimicrobiens sont les $\beta$-lactamines, les céphalosporines et les fluoroquinolones. Chez les animaux, les classes prédominantes étaient les tétracyclines, les $\beta$-lactamines et les « autres antimicrobiens » (figure 1).
Figure 1 : Kilogrammes d'antimicrobiens distribués et/ou vendus pour utilisation chez les animaux et les humains, par classe d'antimicrobiens, en 2014

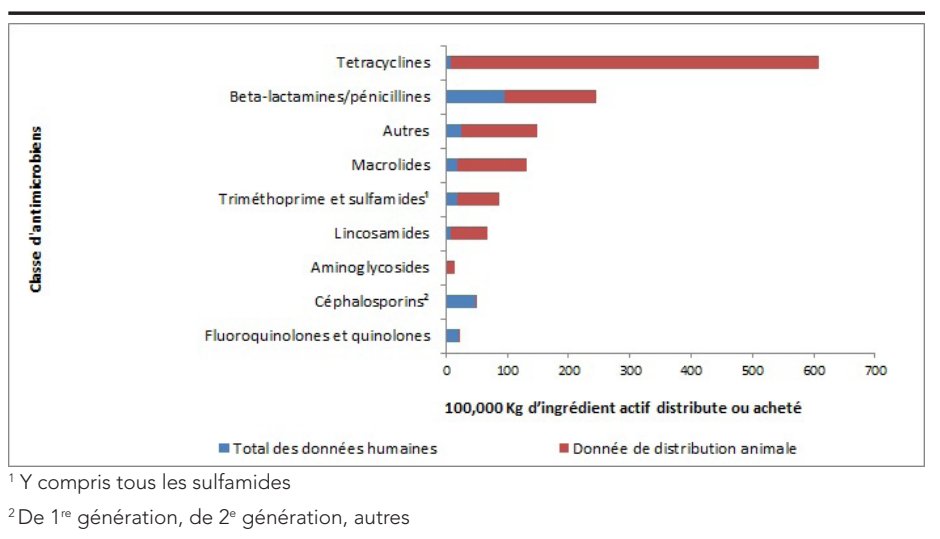

Au cours des cinq dernières années (de 2010 à 2014), 73 \% des antimicrobiens distribués aux animaux destinés à la production alimentaire appartenaient aux mêmes catégories que celles utilisées en médecine humaine; sur ce pourcentage, 1,5 million de kilogrammes d'ingrédients actifs d'antimicrobiens ont été distribués pour utilisation chez les animaux en 2014. II s'agit d'une augmentation de $5 \%$ par rapport à 2013.

\section{Usage chez les humains}

L'utilisation des antimicrobiens chez les humains est demeurée relativement stable au Canada au cours des 13 dernières années. En 2014, par rapport à 2013, il y a eu peu de changements dans la quantité totale d'antimicrobiens distribués par les pharmacies communautaires, tandis que les achats des hôpitaux ont diminué de $4 \%$. La distribution d'antimicrobiens par les pharmacies communautaires représentait $93 \%$ de l'utilisation globale des antimicrobiens. Entre 2010 et 2014, les taux d'ordonnances pour des antimicrobiens ont diminué de $8 \%$ chez les enfants (de 0 à 14 ans) et de $3 \%$ chez les adultes (de 15 à 59 ans), mais on n'a pas observé de diminution chez les personnes âgées ( 60 ans et plus).

En 2014, les hôpitaux canadiens ont acheté 38340 kilogrammes d'antimicrobiens à un coût de 104 millions de dollars. La ciprofloxacine était l'antimicrobien le plus fréquemment achetés en 2014, suivie par l'amoxicilline, l'azithromycine, la ceftriaxone et la doxycycline. Les achats de ceftriaxone et de doxycycline ont connu la plus forte augmentation de 2002 à 2014.

En 2014, la majorité des antimicrobiens utilisés dans la population canadienne de patients externes sont des médicaments administrés par voie orale. Comparativement à 2013, les antimicrobiens parentéraux (intraveineux) les plus souvent administrés en 2014 comprenaient les suivants : daptomycine, ticarcilline et acide clavulanique, fosfomycine, imipénème et cilastatine, ceftazidime, ceftriaxone et colistine. Une augmentation constante a été observée au cours des cinq dernières années dans la distribution des antimicrobiens parentéraux.

En 2014, les antimicrobiens étaient le traitement recommandé dans $8 \%$ de l'ensemble des diagnostics. La majorité des recommandations visaient le traitement des infections respiratoires, y compris les infections des voies respiratoires supérieures, la bronchite et la sinusite aiguë (figure 2). Cette tendance est inquiétante, car les antibiotiques ne sont pas recommandés en traitement de première intention pour la bronchite aiguë ou la sinusite aiguë $(10,11)$. En général, le pourcentage de diagnostics pour lesquels on a recommandé un antimicrobien est demeuré stable. 
Figure 2 : Nombre de diagnostics précis par personne et pourcentage de ces diagnostics accompagnés de recommandations liées à un antimicrobien au Canada, en 2014

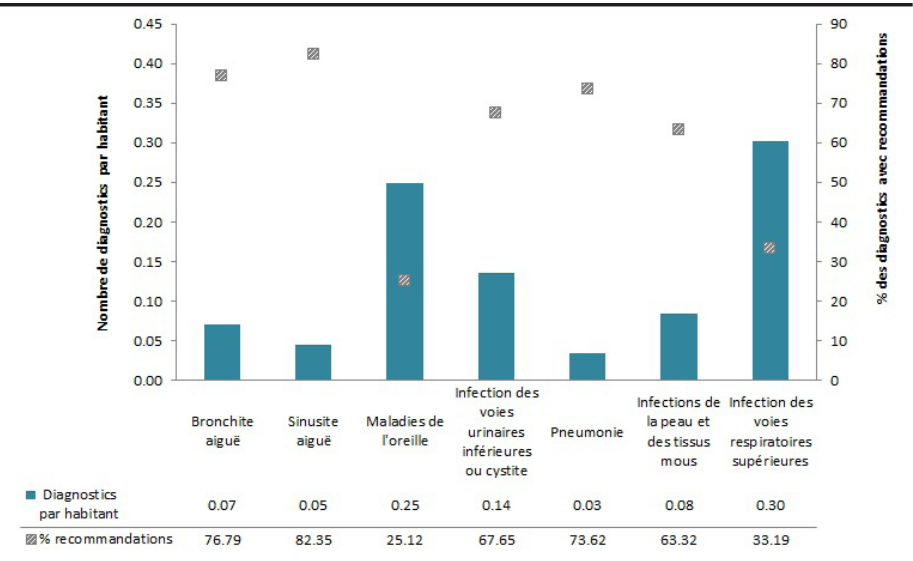

Utilisation chez les animaux destinés à la production alimentaire

La Direction des médicaments vétérinaires de Santé Canada classe les fluoroquinolones dans la catégorie « de très haute importance en médecine humaine ». Depuis 2010, la quantité de fluoroquinolones distribuée à des fins d'utilisation chez les animaux a augmenté de $40 \%$, probablement en raison de l'approbation d'une nouvelle indication du fluoroquinolone. Entre 2013 et 2014, il y a eu une augmentation de 14 \%. Les céphalosporines de troisième génération sont également très importantes pour la médecine humaine. Les données de l'Institut canadien de la santé animale indiquent une diminution de $60 \%$ de la quantité de céphalosporines distribuées pour utilisation chez les animaux de 2011 à 2014.

Comme le montre la figure 3, après ajustement pour tenir compte des chiffres de la population animale et des poids (ligne verte), il y a une augmentation de $3 \%$ depuis 2006 et une augmentation de $1 \%$ depuis 2013 . Au cours des cinq dernières années (2010 à 2014), ce montant a augmenté de $16 \%$.

Figure 3 : Antimicrobiens importants sur le plan médical distribués pour utilisation chez les animaux au fil du temps, mesurés en kilogrammes et en milligrammes d'ingrédients actifs, rajustés en fonction des populations et des poids, de 2006 à 2014

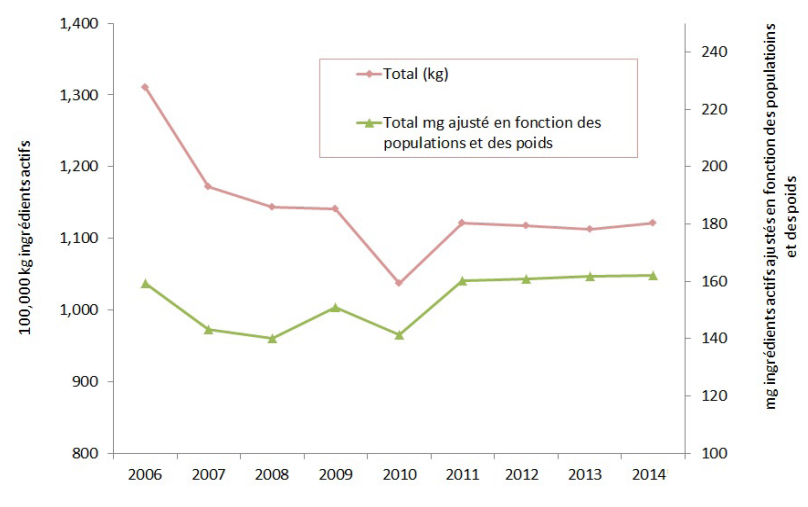

\section{Résistance aux antimicrobiens chez les humains}

\section{Milieux de soins}

Dans les milieux de soins de santé, on assure le suivi des tendances de la résistance aux antimicrobiens pour les organismes ayant priorité absolue (ceux définis comme les plus préoccupants) (tableau 2).

Tableau 2 : Tendances en matière de résistance aux antimicrobiens dans le cas des organismes considérés comme des organismes prioritaires qui sont principalement transmis dans les milieux de soins de santé

\begin{tabular}{|c|c|}
\hline Organisme prioritaire ${ }^{1}$ & $\begin{array}{c}\text { Tendances liées à la résistance aux } \\
\text { antimicrobiens }\end{array}$ \\
\hline $\begin{array}{l}\text { Infection au Clostridium } \\
\text { difficile (ICD) associée aux } \\
\text { soins de santé }\end{array}$ & $\begin{array}{l}\text { Les taux globaux d'infection au Clostridium difficile } \\
\text { associée aux soins de santé ont atteint un sommet en } \\
2008 \text {, soit } 5,8 \text { ICD associées aux soins de santé pour } \\
1000 \text { hospitalisations, puis ils ont diminué lentement } \\
\text { à environ 3,4 ICD associées aux soins de santé pour } \\
1000 \text { hospitalisations en } 2014 \text {. }\end{array}$ \\
\hline $\begin{array}{l}\text { Entérobactéries résistantes aux } \\
\text { carbapénèmes (ERC) }\end{array}$ & $\begin{array}{l}\text { Les taux d'ERC sont restés relativement stables } \\
\text { de } 2010 \text { à } 2014 \text {. En 2014, les taux étaient de 0,22 } \\
\text { pour } 10000 \text { jours-patients, par rapport à 0,19 pour } \\
10000 \text { jours-patients en } 2010 \text {. }\end{array}$ \\
\hline Espèce Enterococcus & $\begin{array}{l}\text { Les taux d'infection à ERV ont fortement augmenté en } \\
\text { passant de } 0,1 \text { cas pour } 10000 \text { jours-patients en } 2007 \text { à } \\
0,61 \text { cas pour } 10000 \text { jours-patients, avant de diminuer } \\
\text { à } 0,45 \text { cas pour } 10000 \text { jours-patients en } 2014 \text {. }\end{array}$ \\
\hline $\begin{array}{l}\text { Staphylococcus aureus } \\
\text { résistant à la méthicilline } \\
\text { (SASRM) }\end{array}$ & $\begin{array}{l}\text { Les infections à SARM ont diminué d'environ } 25 \% \\
\text { depuis } 2008 \text { avec des taux d'infection chutant de } \\
2,92 \text { pour } 1000 \text { hospitalisations en } 2008 \text { à } 2,12 \text { pour } \\
1000 \text { hospitalisations en } 2016 \text {. }\end{array}$ \\
\hline
\end{tabular}
résistance aux antimicrobiens du CDMTI en décembre 2014 (rapport non publié)

\section{Milieux communautaires}

Les tendances en matière de résistance aux antimicrobiens pour les organismes ayant priorité absolue dans les milieux communautaires sont présentées dans le rapport annuel du SCSRA de 2016 (tableau 3).

\section{Tableau 3 : Tendances en matière de résistance} aux antimicrobiens dans le cas des organismes considérés comme des organismes prioritaires qui sont principalement transmis en milieu communautaire

\begin{tabular}{|c|c|}
\hline $\begin{array}{l}\text { Organisme } \\
\text { prioritaire }^{1}\end{array}$ & Tendances liées à la résistance aux antimicrobiens \\
\hline $\begin{array}{l}\text { Neisseria } \\
\text { gonorrhoeae }\end{array}$ & $\begin{array}{l}\text { En } 2014 \text {, on a découvert que } 1995 \text { échantillons de } N \text {. gonorrhoeae au total } \\
(52,4 \% \text { ) étaient résistants à un ou plusieurs antibiotiques, ce qui représentait } \\
\text { une augmentation de la résistance aux antimicrobiens depuis } 2009 \text {, surtout pour } \\
\text { l'azithromycine, la ciprofloxacine, l'éythromycine, la pénicilline et la tétracycline. }\end{array}$ \\
\hline \multirow{2}{*}{$\begin{array}{l}\text { Streptococcus } \\
\text { pyogenes et } \\
\text { S. pneumoniae }\end{array}$} & $\begin{array}{l}\text { La résistance à un certain nombre d'antimicrobiens utilisés pour traiter } \\
\text { Streptococcus pneumoniae a diminué depuis l'introduction du vaccin contre le } \\
\text { pneumocoque en } 2010 \text {. Par exemple, la résistance à la pénicilline a diminué de } \\
12 \% \text { en } 2011 \text { à } 9 \% \text { en } 2014 \text {. }\end{array}$ \\
\hline & $\begin{array}{l}\text { En 2014, tous les échantillons de Streptococcus pyogenes étaient sensibles aux } \\
\text { antimicrobiens de première intention, alors que la résistance aux médicaments de } \\
\text { deuxième intention était stable ou avait diminué depuis } 2010 \text {. }\end{array}$ \\
\hline $\begin{array}{l}\text { Mycobacterium } \\
\text { tuberculosis }\end{array}$ & $\begin{array}{l}\text { Entre } 2004 \text { et } 2014,9 \% \text { des cas à culture positive se sont avérés résistants à au } \\
\text { moins un des antituberculeux de première intention. La résistance à l'isoniazide était } \\
\text { le profil de résistance le plus couramment signalé. De plus, pour la même période, } \\
8 \% \text { des cas à culture positive étaient des cas de monorésistance, } 0,5 \% \text { étaient des } \\
\text { cas de polyrésistance, et } 1 \% \text { étaient des cas de tuberculose multirésistante. De } \\
\text { plus, la proportion de cas de tuberculose multirésistante est demeurée relativement } \\
\text { stable, variant entre } 1 \% \text { et } 2 \% \text {. }\end{array}$ \\
\hline \multirow{2}{*}{$\begin{array}{l}\text { Espèce } \\
\text { Salmonella }\end{array}$} & $\begin{array}{l}82 \% \text { des isolats typhoïdiques étaient résistants à l'acide nalidixique. La résistance } \\
\text { à la ciprofloxacine a augmenté, passant de } 0 \% \text { à } 14 \% \text { de } 2003 \text { à 2014, dont } 16 \% \\
\text { étaient multirésistants. }\end{array}$ \\
\hline & $\begin{array}{l}\text { Au fil du temps, la résistance dans la plupart des isolats non typhoïdiques a diminué } \\
\text { depuis } 2004 \text {, à l'exception de la résistance à l'acide nalidixique, qui a presque } \\
\text { doublé entre } 2013(5 \%) \text { et } 2014(9 \%) \text {. }\end{array}$ \\
\hline
\end{tabular}




\section{Résistance aux antimicrobiens chez les animaux destinés à la production alimentaire}

L'ASPC surveille la résistance aux antimicrobiens de certains organismes bactériens chez plusieurs animaux destinés à la production alimentaire à l'échelle du Canada. Les données de 2014 concernant la résistance aux antimicrobiens chez les animaux destinés à la production alimentaire sont présentées dans le tableau 4

\section{Tableau 4 : Tendances en matière de résistance aux antimicrobiens dans le cas des organismes transmis par les animaux destinés à la production alimentaire}

\begin{tabular}{|c|c|}
\hline $\begin{array}{l}\text { Organisme } \\
\text { prioritaire }^{1}\end{array}$ & $\begin{array}{c}\text { Tendances liées à la résistance aux } \\
\text { antimicrobiens }\end{array}$ \\
\hline Escherichia coli & $\begin{array}{l}\text { En } 2014,96 \% \text { des échantillons de poulet, } 55 \% \text { des } \\
\text { échantillons de porc, et } 56 \% \text { des échantillons de bovins } \\
\text { ont été testés positifs pour l'E. coli générique. À la } \\
\text { suite de l'interdiction de l'utilisation préventive des } \\
\text { antimicrobiens en } 2014, \text { la résistance aux céphalosporines } \\
\text { de troisième génération dans les isolats d'E. coli } \\
\text { provenant d'échantillons de poulet dans l'ensemble de } \\
\text { la chaîne alimentaire a diminué. En } 2014 \text {, seulement } 21 \% \\
\text { des isolats étaient résistants aux céphalosporines de } \\
\text { troisième génération par rapport à } 28 \% \text { en } 2013 \text {. }\end{array}$ \\
\hline $\begin{array}{l}\text { Espèce } \\
\text { Campylobacter }\end{array}$ & $\begin{array}{l}\text { Poulet : } 44 \% \text { des isolats étaient résistants à la tétracycline. } \\
\text { La résistance à la ciprofloxacine a augmenté en passant } \\
\text { de } 4 \% \text { à } 11 \% \text { entre } 2004 \text { et } 2014 \text {. } \\
\text { Porc: Des taux de résistance élevés à la tétracycline } \\
\text { (78\%), à l'azithromycine ( } 53 \% \text { ), à la télithromycine } \\
\text { (42\%) ont été relevés en } 2014 \text {; les taux de résistance à la } \\
\text { cyprofloxacine sont demeurés stable à } 11 \% \text {. } \\
\text { Bovins : La résistance concerne principalement la } \\
\text { tétracycline, avec } 54 \% \text { d'isolats résistants en } 2014 \text {. La } \\
\text { résistance à la ciprofloxacine a augmenté entre } 2008 \text { et } \\
2014 \text {, en passant de } 2 \% \text { à } 7 \% \text { sur cette période. }\end{array}$ \\
\hline Espèce Salmonella & $\begin{array}{l}\text { La résistance à la ceftriaxone des espèces Salmonella } \\
\text { a diminué chez le poulet passant de } 24 \% \text { en } 2013 \text { à } \\
17 \% \text { en } 2014 \text { et aucune résistance n'a été notée à la } \\
\text { ciprofloxacine. Chez le porc, la résistance à la ceftriaxone } \\
\text { des espèces Salmonella est demeurée faible et stable à } \\
4 \% \text {. }\end{array}$ \\
\hline
\end{tabular}

résistance aux antimicrobiens du CDMTI en décembre 2014 (rapport non publié)

\section{Discussion}

Le rapport du SCSRA de 2016 démontre qu'en 2014, plus de $80 \%$ des antimicrobiens distribués au Canada ont été utilisés chez les animaux destinés à la production alimentaire. L'utilisation des antimicrobiens chez les humains et les animaux est demeurée relativement stable au cours des quelques dernières années. Les infections à $C$. difficile associée aux soins de santé, les entérocoques résistants à la vancomycine (ERV), et le Staphylococcus aureus résistant à la méthicilline (SARM), ont diminué, alors que les entérobactéries résistantes aux carbapénèmes (ERC) sont demeurées relativement stables. Au sein de la collectivité, les isolats résistants de S. pneumoniae ont progressivement diminué; les souches de N. gonorrhoeae résistantes aux médicaments ont augmenté, tandis que la tuberculose pharmacorésistante est demeurée stable. Chez les animaux destinés à la production alimentaire, la résistance aux céphalosporines de troisième génération des espèces génériques $E$. coli et Salmonella a diminué chez le poulet, tandis que la résistanceà la ciprofloxacine des espèces Campylobacter à agumenté chez le poulet et le bovin.
Avec la mise en œuvre du SCSRA, l'ASPC a amélioré la coordination mis en place de solides programmes de surveillance pour l'utilisation des antimicrobiens et la résistance aux antimicrobiens en vue d'aider les intervenants par rapport aux données intégrées dont ils ont besoin pour éclairer les nouveaux progrès en termes de réduction de la menace de la résitance aux antimicrobiens et de préservation de l'efficacité des antimicrobiens d'aujourd'hui.

Malgré ces progrès dans notre capacité de surveillance, des lacunes subsistent. Il doit y avoir davantage de données portant sur un certain nombre de pathogènes prioritaires (p. ex. E. coli, Neisseria gonorrhoea et Shigella), et il existe peu de données sur la résistance aux antimicrobiens dans les hôpitaux non universitaires de plus petite taille, y notamment ceux dans des milieux de soins de santé ruraux et du Nord et dans les collectivités des Premières Nations. Dans l'ensemble, il existe peu de données sur la résistance aux antimicrobiens au sein de la collectivité, dans les cliniques de consultation externe, dans les établissements de soins de longue durée, et dans les cabinets de médecins et de dentistes. Les données sur l'utilisation des antimicrobiens chez les animaux sont également limitées. Par exemple, les données sont limitées en ce qui concerne les quantités d'antimicrobiens en vente libre vendus sans ordonnance du vétérinaire, ou les quantités importées comme ingrédients pharmaceutiques actifs destinées aux préparations des vétérinaires et utilisées chez les animaux destinées à la production alimentaire. Même avec toutes les données dont nous disposons, nous ne comprenons toujours pas complètement le lien entre l'utilisation des antimicrobiens et les tendances émergentes de la résistance aux antimicrobiens au Canada.

L'ASPC s'engage à combler ces lacunes en matière de surveillance en travaillant avec les partenaires provinciaux et territoriaux et d'autres intervenants pour élargir les activités de surveillance en vue de recueillir des données de qualité sur les pratiques de prescription des professionnels de la santé, les taux d'infection et les profils de résistance des principaux organismes prioritaires, en particulier en milieu communautaire. I'ASPC travaille aussic avec l'Organisation mondiale de la Santé pour élaborer des indicateurs communs pour des agents pathogènes résistants aux antimicrobiens.

\section{Remerciements}

Les auteurs tiennent à remercier tous les membres du Programme canadien de surveillance des infections nosocomiales, du Programme intégré canadien de surveillance de la résistance aux antimicrobiens, du Système canadien de surveillance des laboratoires de tuberculose, du Système canadien de déclaration des cas de tuberculose, du Système de surveillance de la résistance aux antimicrobiens de Neisseria gonorrhoeae, de la surveillance à l'échelle nationale des maladies invasives à streptocoque, du Programme canadien de surveillance des souches et de la résistance aux médicaments ayant trait au $\mathrm{VIH}$, ainsi que du Laboratoire national de microbiologie. Les auteurs tiennent également à remercier l'équipe du Système canadien de surveillance de la résistance aux antimicrobiens (SCSRA) pour la création du matériel et des figures utilisés dans le présent résumé. 


\section{Conflit d'intérêts}

Aucun.

\section{Financement}

Ce travail a été appuyé par l'Agence de la santé publique du Canada.

\section{Références}

1. World Health Organization [Internet]. Antimicrobial resistance: Global report on surveillance 2014. Geneva: WHO; June 2014. Disponible à l'adresse : http://apps.who. int/iris/bitstream/10665/112642/1/9789241564748_eng. pdf?ua $=1$.

2. O'Neill J [Internet]. Antimicrobial resistance: Tackling a crisis for the health and wealth of nations. London, UK: Review on Antimicrobial Resistance; December 2014. Disponible à I'adresse : https://amr-review.org/sites/default/files/AMR\%20 Review\%20Paper\%20-\%20Tackling\%20a\%20crisis\%20for\%20 the\%20health\%20and\%20wealth\%20of\%20nations_1.pdf.

3. Centers for Disease Control and Prevention [Internet]. National Antimicrobial Resistance Monitoring System for Enteric Bacteria (NARMS). Antibiotic use in food production animals. Atlanta, GA: CDC; 2014. [mis à jour le 29 mars 2016; consulté le 5 oct 2016]. Disponible à l'adresse : https://www.cdc.gov/narms/animals.html.

4. Agence de la santé publique du Canada [Internet]. Système canadien de surveillance de la résistance aux antimicrobiens - Rapport de 2015. Ottawa, ON: ASPC; 2015 mars. Disponible à l'adresse : http://healthycanadians.gc.ca/alt/ pdf/publications/drugs-products-medicaments-produits/ antibiotic-resistance-antibiotique/antimicrobial-surveillanceantimicrobioresistance-fra.pdf?_ga=1.101498113.16728263 05.1472829660.

5. Agence de la santé publique du Canada [Internet]. Système canadien de surveillance de la résistance aux antimicrobiens - Rapport de 2016. Ottawa, ON: ASPC;
2015 mars. Disponible à l'adresse : http://healthycanadians. gc.ca/publications/drugs-products-medicaments-produits/ antibiotic-resistance-antibiotique/alt/pub-fra.pdf.

6. Gouvernement du Canada [Internet]. Rapport sur I'utilisation des antimicrobiens chez les humains - 2014. Guelph ON: Agence de la santé publique du Canada; novembre 2015. [mis à jour le 17 nov 2015; consulté le 5 oct 2016]. Disponible à l'adresse : http://healthycanadians.gc.ca/ publications/drugs-products-medicaments-produits/humanantimicrobial-use-2014-utilisation-antimicrobiens-humains/ index-fra.php.

7. Agence de la santé publique du Canada [Internet]. Surveillance des microorganismes résistants aux antimicrobiens (MRA) : Rapport sommaire d'après les données du 1er janvier 2009 au 31 décembre 2014. Ottawa, ON: ASPC; 2015 août 2015. [mis à jour le 29 sept 2016; consulté le 5 oct. 2016]. Disponible à l'adresse : http:// healthycanadians.gc.ca/publications/drugs-productsmedicaments-produits/antimicrobial-summary-sommaireantimicrobien/index-fra.php.

8. Agence de la santé publique du Canada [Internet].PICRA 2013 - Rapport annuel : Chapitre 1. Design et Méthodes. Ottawa, ON: ASPC; 2014. Disponible à l'adresses : http:// www.phac-aspc.gc.ca/cipars-picra/2013/annu-reportrapport-fra.php.

9. Agence de la santé publique du Canada [Internet]. La tuberculose au Canada 2014 - Prédiffusion. Ottawa, ON: ASPC; 2016 juin. Disponible à l'adresse : http:// healthycanadians.gc.ca/publications/diseases-conditionsmaladies-affections/tuberculosis-2014-tuberculose/index-fra. php\#a3.

10. Alberta Medical Association [Internet]. Guidelines for the management of acute bronchitis. 2008 update. Edmonton, AB: Alberta Medical Association; 2008. Disponible à I'adresse : http://www.topalbertadoctors.org/download/378/ acute_bronchitis_guideline.pdf.

11. Rosenfield RM, Picirrilo JF, Chandrasekhar SS, et al. Clinical practice guideline update: Adults sinusitis executive summary. Otolaryngol Head Neck Surg. 2015 Apr;152(4):598-609. 